\title{
Isolated pulmonary arteriovenous malformations requiring anatomic resection
}

\author{
Michael J. Mann, MD, ${ }^{a}$ Miranda J. Kramer, RN, MS, ${ }^{a}$ Timothy S. Hall, MD, ${ }^{a}$

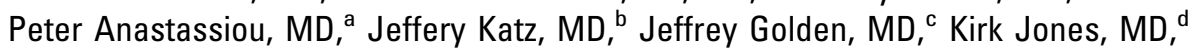 \\ and David M. Jablons, MD, ${ }^{\text {a }}$ San Francisco, Calif
}

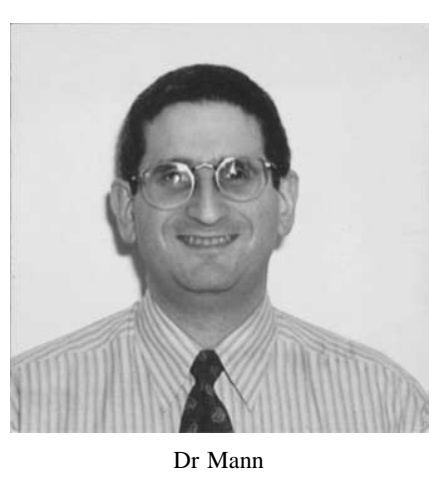

$\mathrm{P}$ ulmonary arteriovenous malformations (AVMs) are fistulous communications that are often part of a generalized syndrome and that can lead either to shunting or to hemoptysis. Identification and anatomic localization may allow selective embolization. Although this strategy can preserve lung parenchyma, resection may still be necessary to achieve adequate treatment of life-threatening complications. We present 2 cases of isolated pulmonary AVMs in which either the extent of disease or the need for prompt definitive treatment led to anatomic resection.

\section{Clinical Summaries}

PATIENT 1. A 41-year-old woman with hemoptysis had undergone ligation of a persistent ductus arteriosus via a left thoracotomy at age 28. Chest computed tomography (CT) and bronchoscopy failed to identify the source of bleeding. She was hospitalized 1 month later with recurrent hemoptysis and underwent repeat CT and bronchoscopy, which did not localize the site of bleeding. Further evaluation of the right bronchial arteriography did not reveal evidence of a vascular abnormality. The left bronchial arteriography could not be assessed. The right bronchial vessels were embolized empirically, and the patient was discharged after a period of uneventful observation. She returned later that day, however, with hemoptysis that necessitated emergency isolation of the left lung with a double-lumen endotracheal tube. Repeat CT revealed a large amount of blood in the left pulmonary parenchyma and airways, with a possible focus of hemorrhage in the superior segment of the left lower lobe (Figure 1). Arterial blood gas, complete blood count, pro-time, partial thromboplastin time, and liver function test results were normal. Additional attempts at pulmonary and bronchial angiography failed to yield a source of hemorrhage. The patient was brought to the operating room for exploration, repair, or resection.

\footnotetext{
From the Divisions of Cardiothoracic Surgery, ${ }^{\mathrm{a}}$ Thoracic Anesthesia, ${ }^{\mathrm{b}}$ Pulmonary Medicine, ${ }^{\mathrm{c}}$ and Pulmonary Pathology, ${ }^{\mathrm{d}}$ University of California, San Francisco, Calif.

Received for publication Sept 3, 2003; revisions requested Sept 30, 2003; accepted for publication Oct 8, 2003.

Address for reprints: Michael J. Mann, MD, Division of Cardiothoracic Surgery, UCSF Medical Center, 505 Parnassus Ave, Room M-593, San Francisco, CA 94143 (E-mail: mannm@ surgery.ucsf.edu).

J Thorac Cardiovasc Surg 2004;127:574-6

$0022-5223 / \$ 30.00$

Copyright $\odot 2004$ by The American Association for Thoracic Surgery

doi:10.1016/j.jtcvs.2003.09.031
}

At operation, a frontal, transsternal left hemiclamshell (fourth intercostal space) approach was chosen to allow proximal control of the pulmonary artery and access for cardiopulmonary bypass. Thoracotomy, however, revealed only evidence of acute and subacute hemorrhage in the left lung. The area of the previous patent ductus arteriosus ligation was carefully dissected, and no evidence of a fistula was found. The left bronchial lumen of the endotracheal tube was withdrawn to allow intraoperative bronchoscopy of the distal trachea and left main stem bronchus; a moderate amount of clot was present in an otherwise grossly normal-appearing airway. At this juncture, staged resection of the left lung was performed. After left lower lobectomy, the specimen was carefully dissected on the back table and examined for a source of bleeding. A small area of bronchiectasis was identified in the superior segment; this abnormality alone was not believed to provide an adequate explanation for the patient's massive hemorrhage. Given the patient's recent life-threatening hemoptysis, a completion pneumonectomy was performed. The patient was extubated immediately after surgery, had an uneventful recovery, and was discharged home on postoperative day 10 with no subsequent episodes of bleeding. At 6 months, the patient remained asymptomatic, with an excellent performance status. Pathologic examination revealed a $1-\mathrm{cm}$ AVM that involved the distal left mainstem bronchus; it was characterized by thickened submucosal bronchial arteries with associated ectatic blood vessels and recent and remote hemorrhage (Figure 2).

PATIENT 2. Marked cyanosis, clubbing, and progressive dyspnea on exertion developed in a 19-year-old man without any significant medical history after he moved to an altitude of 7400 feet. CT of the chest revealed numerous anomalous vascular structures in the right lower lobe (Figure 3, A). Pulmonary arteriography confirmed numerous AVMs that involved much of the lower lobe of the right lung (Figure 3, B). Bronchoscopy was unremarkable. His room air arterial blood gas demonstrated an arterial oxygen saturation of $84 \%$, and a screening laboratory complete blood count showed polycythemia with a hematocrit of $65 \%$.

The patient underwent a limited muscle-sparing right thoracotomy, which revealed a vascular abnormality in the right lower lobe but no anomalous vessels in the inferior pulmonary ligament or pulmonary sequestration. Right lower lobectomy was therefore undertaken without event. It is interesting to note that initial dissection in the main fissure with isolation and temporary occlusion of the pulmonary artery to the lower lobe confirmed the presence of the lower lobe shunt. Pathologic examination revealed a $6 \times 6 \times 3 \mathrm{~cm}$ lesion with multiple thin-walled, dilated vessels (Figure 4). The patient was dis- 


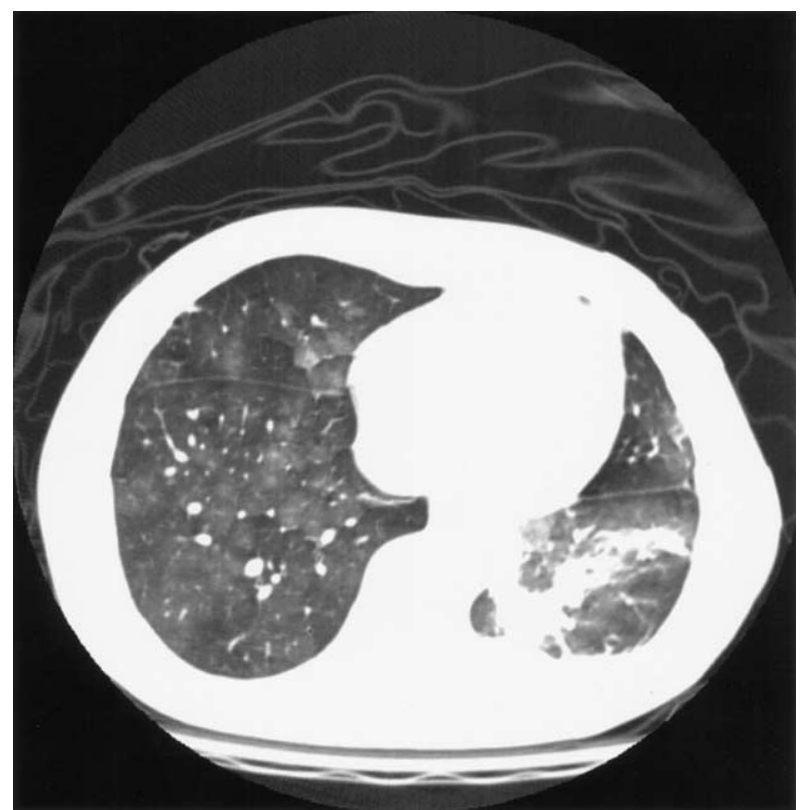

Figure 1. Chest computed tomography scan of a 41-year-old woman with recurrent hemoptysis. Diffuse hemorrhage is noted throughout the lower lobe of the left lung without a clear source of bleeding.

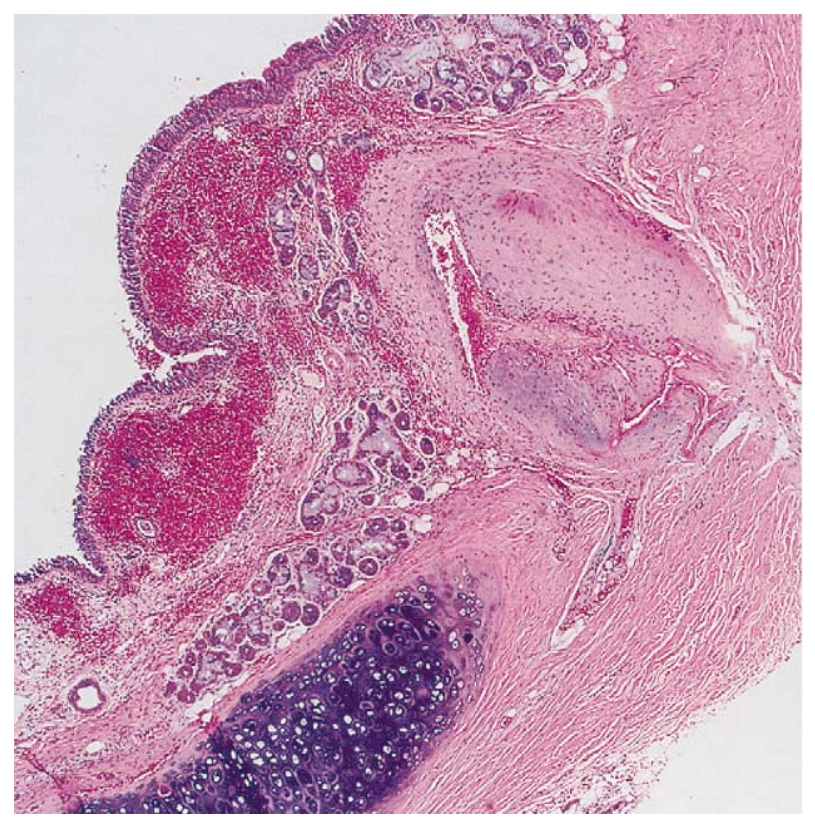

Figure 2. Histologic examination of a 1-cm submucosal AVM involving the distal left main stem bronchus. Thickened bronchial arteries are seen associated with ectatic blood vessels and recent and remote hemorrhage (stain, hematoxylin and eosin; magnification, $40 \times$ ).

charged on postoperative day 4 and remained symptom free after returning to his home at 7400 feet on postoperative week 6. The postoperative hematocrit decreased from $65 \%$ to $52 \%$.
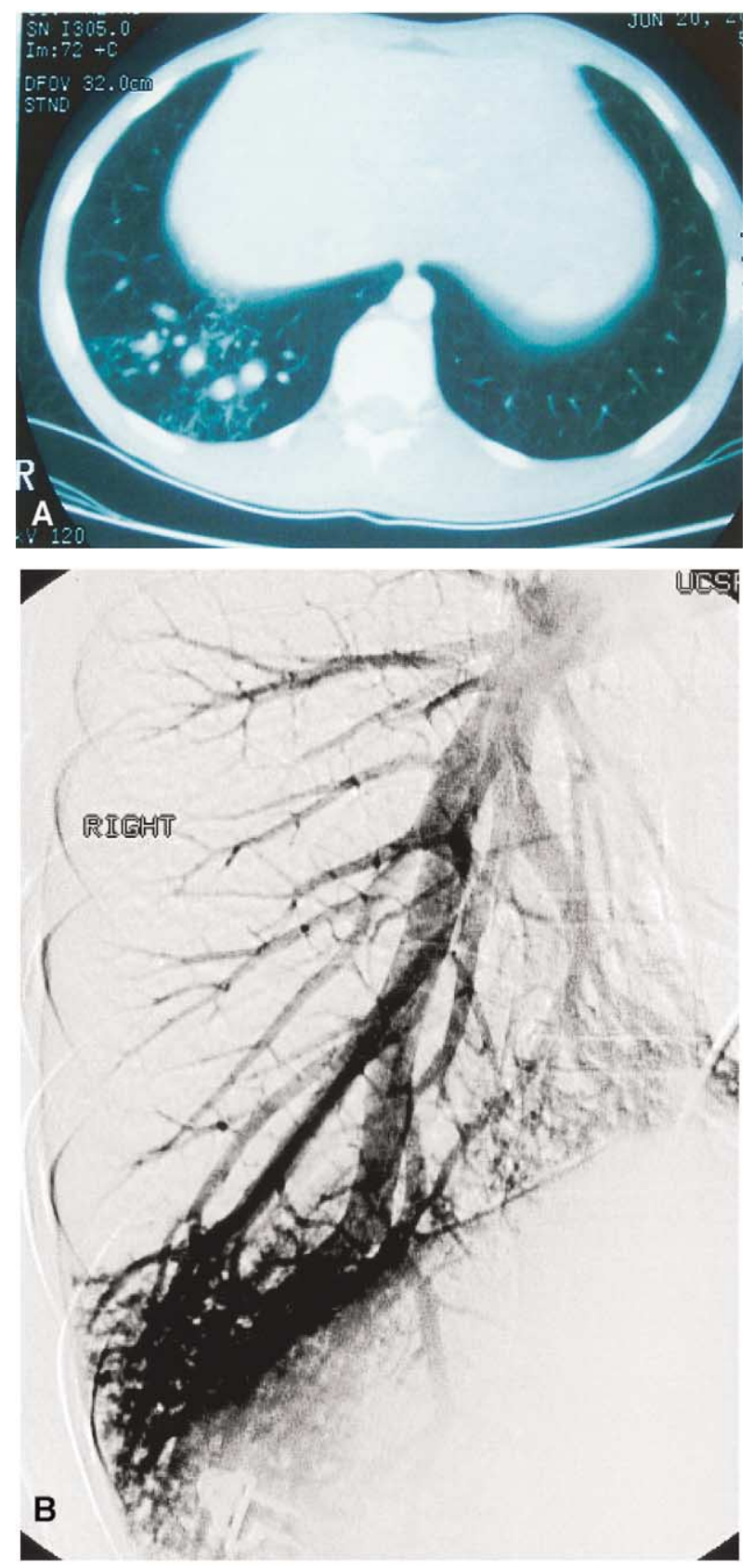

Figure 3. A, Chest computed tomography scan of a 19-year-old man with progressive dyspnea at high altitudes. Dense vascular markings in the right lower lobe appear consistent with atrioventricular malformations. $B$, Pulmonary angiography reveals the characteristic blush of multiple large atrioventricular malformations in the right lower lobe.

\section{Discussion}

Pulmonary AVMs were first described in the literature by Wilkins ${ }^{1}$ in 1918. This entity affects women twice as often as men, involves the lower lobes in most cases, ${ }^{2}$ and in $50 \%$ of cases is found in conjunction with arteriovenous fistulas in other anatomic loca- 


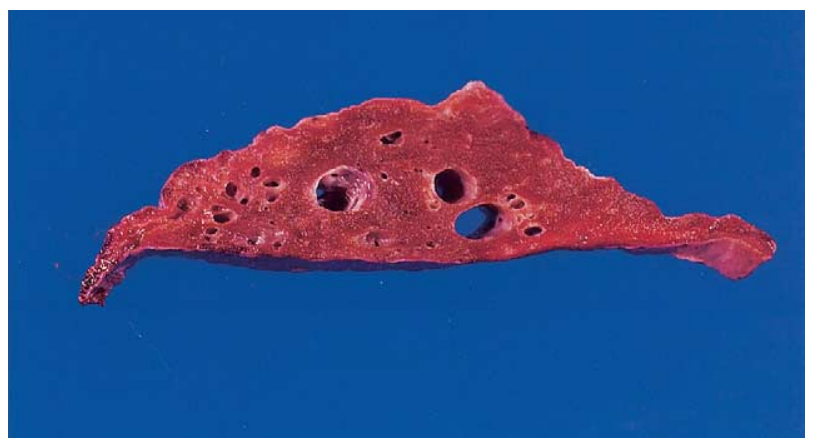

Figure 4. Cross section of the right lower lobe reveals numerous abnormally located, ectatic vessels within the pulmonary parenchyma.

tions. $^{3-5}$ It has been associated with congenital failure of arteriovenous septation, and an associated vascular defect may lead to progressive AVM enlargement. ${ }^{2,4,5}$ Such enlargement over time may explain the relatively late presentation of most of these lesions. It has been postulated that hypoxemia can lead to a progressive increase of pulmonary vascular resistance in these patients, either because of microthrombi and the increased viscosity associated with polycythemia or because of pulmonary arteriolar constriction, with resultant pulmonary hypertension preferentially increasing flow through the AVM. ${ }^{6}$ Concomitant lesions, such as mitral stenosis, may also contribute to pulmonary hypertension and thereby exacerbate an $\mathrm{AVM}^{7}{ }^{7}$ In the first case presented here, closure of a patent ductus arteriosus may have subsequently encouraged increased AVM flow, and in the second case, a decrease in environmental oxygen tension exacerbated AVM-related cyanosis.

Although the most common chronic manifestations of pulmonary AVM are dyspnea, clubbing, and cyanosis, a number of acute life-threatening complications can occur. Particularly common are paradoxical emboli leading to systemic embolization; involvement of the cerebral circulation can cause transient ischemic attacks, stroke, or cerebral abscess. ${ }^{8}$ Pulmonary AVM can also present as spontaneous hemothorax or massive hemoptysis, as in our first patient, in whom the AVM had not caused hypoxemia or hemodynamic complications.

Once symptoms or complications manifest, invasive treatment of pulmonary AVM is warranted. Surgical excision can be curative if the entire lesion can be safely and completely resected. ${ }^{9,10}$ Percutaneous embolization of pulmonary AVMs has become established as an effective means of preserving lung parenchyma, particularly in poor surgical candidates. ${ }^{11-14}$ Although lung-sparing embolization was attempted several times in our first case, pneumonectomy was ultimately required to save this otherwise healthy patient's life. Alternatively, in our second case, diffuse disease isolated to the right lower lobe rendered lobectomy the procedure of choice. As with any rare disease, successful treatment of the complications of pulmonary AVM first requires diagnosis. Although modern radiologic techniques offer some patients an opportunity for minimally invasive treatment, a long-term solution that optimally reduces the risk of mortality while preserving functional capacity remains the primary goal of therapy and should determine the ultimate course of intervention.

These cases of pulmonary AVM demonstrate both a common (lower lobe pulmonary artery) and a rare (bronchial submucosal) variant and their attendant symptomatology. Successful management of these patients mandates close interaction and communication among pulmonologists, interventional and diagnostic radiologists, and surgeons. Although pulmonary AVMs are rare, they should be included in any differential diagnosis of worsening dyspnea, stroke, or hemoptysis. They can present as isolated pulmonary/bronchial anomalies or as part of a systemic syndrome. Prompt workup and intervention can lead to dramatic improvement in symptoms and, in the setting of hemoptysis, can be life-saving.

\section{References}

1. Wilkens GP. Ein Fall von Multiplen Pulmonalis Aneurysmen. Beitr $z$ Klin d Tuberk. 1918;38:1.

2. Dines DE, Arms RA, Bernatz PE, Gomes MR. Pulmonary arteriovenous fistulas. Mayo Clin Proc. 1974;49:460-5.

3. Goldman A. Arteriovenous fistula of the lung: its hereditary and clinical aspects. Am Rev Tuberc. 1948;57:266-80.

4. Gomes MR, Bernatz PE, Dines DE. Pulmonary arteriovenous fistulas. Ann Thorac Surg. 1969;7:582-91.

5. Logan WD Jr, Crispin RH. Hereditary hemorrhagic telangiectasia with pulmonary arteriovenous fistula. South Med J. 1962;55:29-37.

6. LeRoux BT. Pulmonary arteriovenous fistulae. $Q$ J Med. 1959;28:120.

7. Chow LT, Chow W, Ma K. Pulmonary arteriovenous malformation: progressive enlargement with replacement of the entire right middle lobe in a patient with concomitant mitral stenosis. Med J Aust. 1993; 158:632-4.

8. Hodgson CH, Kaye RL. Pulmonary arteriovenous fistula and hereditary hemorrhagic telangiectasia: a review and report of 35 cases of fistula. Dis Chest. 1963;43:449-55.

9. Bosher LH Jr, Blake DA, Byrd BR. An analysis of the pathologic anatomy of pulmonary arteriovenous aneurysms with particular reference to the applicability of local excision. Surgery. 1959;45: 91-100.

10. Hudspeth AS, Canipe TL. Pulmonary arteriovenous fistula: operative pulmonary venous blood gas studies as an aid to resectional therapy. Ann Thorac Surg. 1965;1:641-5.

11. Hughes JMB, Allison DJ. Pulmonary arteriovenous malformations: the radiologist replaces the surgeon. Clin Radiol. 1990;41:297-8.

12. Castaneda-Zuniga W, Epstein M, Zollikofer C, Nath PH, Formanek A, Ben-Shachar G, et al. Embolization of multiple pulmonary artery fistulas. Radiology. 1980;134:309-12.

13. Hatfield DR, Fried AM. Therapeutic embolization of diffuse pulmonary arteriovenous malformations. AJR Am J Roentgenol. 1981; 137:861-5.

14. Wallenhaupt SL, D'Souza V. Combined radiological and surgical management of arteriovenous malformation of the lung. Ann Thorac Surg. 1988;45:213-5. 